\title{
Un desafío clínico: diagnóstico diferencial de pancreatitis autoinmune y cáncer de páncreas. El valor del tratamiento esteroidal de prueba
}

\author{
ZOLTÁN BERGER PhD ${ }^{1,2}$
}

\section{Focal autoimmune pancreatitis versus pancreatic cancer: value of steroid treatment in the diagnosis}

Background: The differential diagnosis of pancreatic cancer and focal forms of autoimmune pancreatitis is complicated since serological tests, IgG4 and CA 19-9 have a low sensibility and specificity. CT scan and magnetic resonance imaging provide clear differentiation in the majority, but not in all cases. Endosonography is the most precise diagnostic procedure and allows to obtain samples for cytology or even histological studies. Aim: To report the experience with 18 cases of focal autoimmune pancreatitis and three cases of pancreatic cancer. Material and Methods: Review of medical records of 18 patients with focal autoimmune pancreatitis and 3 cases of pancreatic cancer. Results: The eighteen patients with focal autoimmune pancreatitis were treated with prednisone $0.5 \mathrm{mg} / \mathrm{kg} /$ day obtaining a complete clinical and morphological recovery in all. However, 3 had a relapse and one was operated. During follow up, none has developed a pancreatic cancer. The 3 patients with pancreatic cancer did not respond to steroidal treatment. Conclusions: The quick and dramatic response to steroids of autoimmune pancreatitis, may be useful and is recommended for the differential diagnosis with pancreatic cancer.

(Rev Med Chile 2014; 142: 413-417)

Key words: Autoimmunity; Pancreatitis, acute necrotizing; Pancreatic neoplasms.

U na forma particular de la pancreatitis inflamatoria fue descrita por la primera vez por Henri Sarles en $1961^{1}$, pero el concepto de autoinmunidad comienza a ser aceptado desde $1995^{2}$. Ya se describieron dos formas histopatológicas de la enfermedad ${ }^{3,4}$ : El tipo 1, la pancreatitis esclerosante linfoplasmocítica (PELP) es la forma originalmente descrita, considerado como parte de una enfermedad sistémica IgG4 dependiente, acompañada por niveles séricos elevados de IgG4, asociada con frecuencia con manifestaciones autoinmunes en otros órganos, en particular colangitis esclerosante primaria IgG4

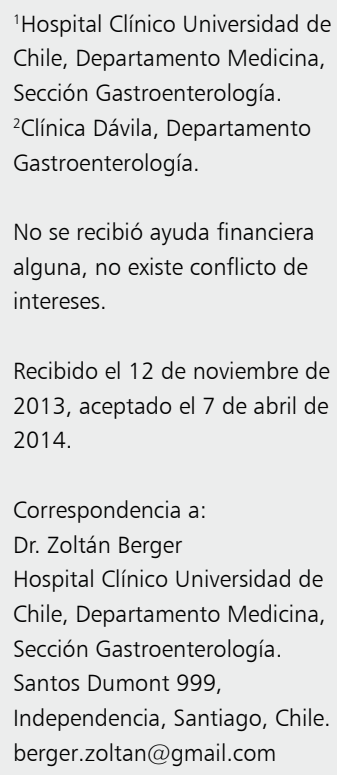

dependiente, inflamación de las glándulas salivales etc. El hallazgo histopatológico del páncreas es la infiltración linfoplasmocitaria por células positivas a IgG4, fibrosis, venulitis obliterante. El tipo 2, la pancreatitis idiopática conducto-destructiva (PICD) está caracterizado clínicamente como enfermedad de páncreas, que se asocia sólo con enfermedad inflamatoria intestinal, el nivel sérico de IgG4 es normal y el examen histológico demuestra lesiones granulocíticas epiteliales, con destrucción de los conductos. Existe también una clasificación clínica propuesta en Italia, que distingue las formas difusas y focales ${ }^{5,6}$, inde- 
pendientemente de la variante histológica. En la práctica clínica cotidiana la diferenciación entre cáncer de páncreas $(\mathrm{CaPa})$ y una forma focal de la pancreatitis autoinmune (PAI) no siempre es fácil. Definir esta incertidumbre diagnóstica no sólo es difícil para el radiólogo, sino representa un gran desafío para el clínico. Por una parte, el desconocimiento de la PAI ha sido un factor importante en la realización de intervenciones quirúrgicas innecesarias. Desde el crecimiento exponencial de las publicaciones sobre la PAI en los últimos años, el número de resecciones pancreáticas con hallazgo definitivo de patología benigna parece estar en disminución. Aun así, en Estados Unidos de Norteamérica (EE.UU.) en 2,5\% de las resecciones pancreáticas realizadas con diagnóstico de $\mathrm{CaPa}$, el hallazgo histológico definitivo es $\mathrm{PAI}^{7}$. Siguen existiendo casos cuando el médico tratante toma la decisión quirúrgica a pesar de no tener certeza en el diagnóstico de la patología maligna, pero tampoco puede descartarla fehacientemente. Por otra parte, el sobrediagnóstico de la PAI resulta en "tratamiento" de CaPa con esteroides, perdiendo el tiempo para realizar cirugía resectiva con intención curativa, que mejora significativamente la sobrevida de estos pacientes.

En este artículo analizamos la evolución clínica con o sin cirugía en nuestros pacientes portadores de PAI focal y el rol de la terapia esteroidal de prueba en la toma de decisiones clínicas.

\section{Pacientes y Métodos}

En nuestro centro tenemos registrados 34 pacientes portadores de PAI en los últimos 15 años. Su historia clínica fue revisada y analizada en forma retrospectiva, aprobado por el Comité de Ética del Hospital Clínico Universidad de Chile. Dieciocho de ellos presentaron la forma focal de la enfermedad, y 8 fueron operados. En los últimos 4 años, tomamos decisión quirúrgica en 3 pacientes, al no poder establecer el diagnóstico de $\mathrm{CaPa}$ y ante la ausencia de respuesta a prednisona. Todos los pacientes fueron sometidos a exámenes radiológicos, tomografia computada (TAC) con contraste y resonancia magnética (RM) también con contraste. En algunos casos se realizó también endosonografía (EUS) con toma de muestra con aguja fina. IgG4 fue solicitado en la mayoría de los pacientes $(n=13)$, el resultado de CA 19-9 fue accesible sólo en algunos de ellos $(\mathrm{n}=5)$.

\section{Resultados}

De nuestros 18 pacientes portadores de PAI focal, 11 no fueron operados inicialmente. Uno de ellos fue estimado por su cirujano como $\mathrm{CaPa}$ inoperable con invasión de vasos grandes, y enviado a nuestro centro para tratamiento endoscópico paliativo. Todos estos pacientes recibieron tratamiento con prednisona, iniciando $0,5 \mathrm{mg} /$ $\mathrm{kg} /$ día y reduciendo la dosis según lo descrito en la literatura. Todos ellos se recuperaron completamente según criterios clínicos y morfológicos, observándose recaída en 3 de ellos, llevándonos tomar decisión quirúrgica en uno de ellos. Los pacientes fueron controlados por más de un año, con la excepción de la última paciente diagnosticada y tratada 3 meses atrás. Ninguno desarrolló un cáncer de páncreas.

Siete pacientes fueron operados en el momento de la duda diagnóstica: en un caso el cirujano, aun por los hallazgos intraoperatorios estimó que el tumor era infiltrante, imposible a resecarlo, sólo realizó anastomosis biliodigestiva paliativa. La indicación quirúrgica de otros 6 pacientes fue la imposibilidad de descartar $\mathrm{CaPa}$, la mayoría de ellos hace algunos años atrás, cuando sólo tuvimos nuestras experiencias iniciales. Un paciente presentó una forma excepcional: un año después de un primer episodio PAI recuperada completamente, recaída de PAI con múltiples lesiones quísticas. Una de ellas respondió parcialmente y se tomó decisión quirúrgica, después de haber conversado con el paciente. La histología de la lesión finalmente comprobó que fue parte de la enfermedad autoinmune, sin sospecha de malignidad.

No hay diferencia significativa en edad del inicio de la enfermedad entre los pacientes operados y no operados, IgG4 fue normal en la mayoría de nuestros casos, el nivel elevado es sólo excepción en nuestra experiencia. Sin embargo, la histología fue compatible en todos los casos resecados con tipo 1 de PAI, ningún caso era compatible con tipo 2. Es decir, el valor diagnóstico de IgG4 en nuestra experiencia ha sido aun mucho menor que en la mayoría de literatura.

La lesión focal comprometió la cabeza de páncreas en 7 de 8 casos operados con ictericia obstructiva consecutiva en 5 de ellos, mientras en el grupo no operado la distribución es más dispersa. La última operación con sospecha de CaPa fue realizada en 2007 en este grupo de pacientes, los 
Pancreatitis autoinmune versus cáncer - Z. Berger

Tabla 1. Características de pacientes portadores de pancreatitis autoinmune focal

\begin{tabular}{|c|c|c|c|}
\hline & Operados & No operados & Total \\
\hline Género (H/M) & $7 / 1$ & $8 / 2$ & $15 / 3$ \\
\hline $\begin{array}{l}\text { Edad } \\
(\overline{\mathbf{x}}, \text { rango })\end{array}$ & $\begin{array}{c}45,25 \\
(21-56)\end{array}$ & $\begin{array}{c}42,4 \\
(12-67)\end{array}$ & $\begin{array}{c}43,67 \\
(12-67)\end{array}$ \\
\hline \multicolumn{4}{|l|}{ Foco } \\
\hline Cabeza & 7 & 3 & 10 \\
\hline Cuerpo & - & 2 & 2 \\
\hline Cola & 1 & 1 & 2 \\
\hline Múltiples & - & 4 & 4 \\
\hline \multicolumn{4}{|l|}{$\lg G 4$} \\
\hline Elevada & $1 *$ & 1 & 2 \\
\hline Normal & 3 & 8 & 11 \\
\hline No & 4 & 1 & 5 \\
\hline $\begin{array}{l}\text { Seguimiento } \\
\text { (meses) }\end{array}$ & $\begin{array}{c}108,75 \\
(36-182)\end{array}$ & $\begin{array}{c}18,4 \\
(3-40)\end{array}$ & $\begin{array}{c}58,56 \\
(3-182)\end{array}$ \\
\hline
\end{tabular}

*Operado con diagnóstico PAI por respuesta parcial de lesión quística.

casos nuevos posteriores fueron tratados con éxito con prednisona y seguidos hasta la recuperación completa (Tabla 1).

Por otra parte, en los últimos 3 años vimos 3 pacientes, cuando las imágenes de TAC y RM no permitieron establecer un diagnóstico certero de $\mathrm{CaPa}$. Surgió la posibilidad de PAI atípica, formando una lesión focal pequeña con densidad alterada, pero sin dilatación muy marcada del conducto pancreático principal, sin evidencia morfológica de un $\mathrm{CaPa}$. Uno de estos pacientes fue sometido a EUS y estudio citológico, sin comprobar lesión maligna, observando sólo presencia de abundantes linfocitos. En los 3 casos iniciamos tratamiento de prueba con prednisona con control de imagen en 2 semanas. En ningún caso encontramos cambio significativo en la imagen, los 3 pacientes fueron sometidos a cirugía - logrando resección en 2 de ellos.

\section{Discusión}

El cáncer de páncreas es uno de los tumores malignos más letales, su diagnóstico es tardío en muchos casos. Su pronóstico es mejor si el tumor es menor, permite una resección quirúrgica curativa, completa en sentido oncológico ${ }^{8}$. El diagnóstico en esta etapa inicial es difícil y -si la imagen radiológica no es típica- surge la posibilidad de existencia de enfermedad benigna de páncreas que puede imitar el cuadro clínico y las alteraciones morfológicas. Cabe enfatizar que esta duda no existe en la mayoría de los casos: por una parte, el cáncer de páncreas es una masa hipovascular y provoca una dilatación importante del conducto pancreático principal acompañada por atrofia del parénquima de este segmento. Por otra parte, el diagnóstico de las enfermedades benignas es también factible en muchos casos, sin tener la duda diagnóstica de cáncer. La "pancreatitis de surco" (groove pancreatitis) $)^{9}$ es una enfermedad muy rara, casi excepcional, su tratamiento endoscópico puede ser exitoso, pero requiere cirugía con cierta frecuencia. La principal alternativa es la pancreatitis autoinmune, con su respuesta dramática al tratamiento por esteroides que permite evitar operaciones con todos sus riesgos asociados.

El diagnóstico de PAI se basa en diferentes criterios. Aunque en la mayoría el diagnóstico es claro con el cuadro clínico, serológico y las imágenes, quedan casos dudosos. Según nuestra experiencia, similar a las publicaciones internacionales, el cuadro clínico no es típico, siendo los hallazgos más frecuentes la ictericia, dolor, baja de peso, diabetes de inicio reciente. La asociación con otras enfermedades autoinmunes, en particular con colangitis esclerosante ${ }^{4,10}$, inflamación de glándulas salivales o enfermedad inflamatoria intestinal puede apoyar PAI. El valor de la serología, IgG4 elevado es variable en la literatura, con valores extremos entre $15-76 \%{ }^{11}$. Encontraron alta sensibilidad y especificidad en publicaciones orientales $^{12,13}$, mucho menos en Europa y EE.UU ${ }^{14}$. Nuestras experiencias son similares a las europeas y a la sensibilidad más baja publicada en la literatura: IgG4 fue alta sólo en 2 casos de 13 PAI (Tabla 1). En la práctica cotidiana, las imágenes tienen el mayor rendimiento en el diagnóstico de la PAI. La alteración típica en la forma difusa es el aumento de tamaño de la glándula con un halo ("imagen de salchicha"), sin colecciones peripancreáticas y sin dilatación del conducto principal, combinación de hallazgos que la distingue claramente de la pancreatitis aguda y de la reagudización de pancreatitis crónica. La gran dificultad es la forma focal: sólo mínima dilatación del conducto principal o ausencia completa de dilatación es la diferencia principal comparando con el cáncer. Lógicamente la herramienta ideal 
sería la biopsia. Sin embargo, su rendimiento es variable, depende de la calidad de la muestra, de la vía de obtenerla y también existe el error de muestreo. Por consiguiente, el valor predictivo negativo de la biopsia en relación con el $\mathrm{CaPa}$ es relativamente bajo. Por otra parte, el diagnóstico histológico certero de la PAI es raramente posible desde las muestras citológicas tomadas por aguja fina, siendo mejor el rendimiento de la biopsia mayor, obteniendo tejido con aguja trucut ${ }^{15}$. El rendimiento de la citología de muestras tomadas durante colangiografía retrógrada endoscópica (ERCP) por cepillado especial es variable, tampoco supera $70 \%{ }^{16}$. Efectivamente, en uno de nuestros casos anteriores logramos realizar EUS y obtener citología, que no detectó células malignas, sólo presencia de abundantes linfocitos, orientándonos más bien hacia el diagnóstico de PAI.

La PAI responde al tratamiento con prednisona, la recuperación casi siempre es completa. Es una enfermedad autoinmune, en la cual se puede suspender el tratamiento y la recaída no es superior de $30 \%$, además, responde igual a nuevo tratamiento. Cuando el diagnóstico es claro, se recomienda controlar la imagen en 8-12 semanas para constatar la recuperación completa. La situación es diferente si hay la más mínima duda en la naturaleza de la enfermedad. La respuesta dramática y rápida a prednisona puede ser utilizada también en el diagnóstico diferencial y forma parte de criterios diagnósticos HISORt aceptados ampliamente (histología, imágenes, serología, otros órganos involucrados, respuesta al tratamiento) ${ }^{17}$.

La respuesta al tratamiento con esteroides es una característica de todas las formas de la PAI, sea tipo histológico 1 ó 2 o según la clasificación clínica, forma focal o difusa. Este tratamiento permite una recuperación completa con riesgo variable de recaída. En la literatura sólo encontré un reporte de caso cuando PAI no respondió en forma satisfactoria al tratamiento con esteroides $^{18}$, en este caso la base de diagnóstico fue sólo la positividad tardía de anticuerpo antinuclear, criterio diagnóstico por lo menos discutible. No hay observación de asociación de PAI con $\mathrm{CaPa}$, no hay riesgo de malignización de la enfermedad. Por consiguiente, el diagnóstico correcto de PAI permite evitar operaciones innecesarias, con todos sus riesgos inmediatos y consecuencias posteriores, como riesgo de insuficiencia exo y endocrina del páncreas. Efectivamente, en 7 de nuestros 8 pacientes operados, un tratamiento de prueba con prednisona habría descartado la sospecha de cáncer y evitado la cirugía. Sólo en un paciente tomamos decisión quirúrgica, conversando también con él, sabiendo el diagnóstico, pero observando sólo respuesta parcial de una de las lesiones quísticas al tratamiento. Aun en su caso, la continuación de la terapia podría haber sido una alternativa. Por otro lado, ausencia de respuesta a esteroides prácticamente descarta PAI. En el trabajo de Moon et al ${ }^{19}$, de los 22 pacientes estudiados los 15 que respondieron fueron portadores de PAI y se recuperaron completamente con la continuación del tratamiento, mientras los 7 no respondedores fueron invariablemente portadores de $\mathrm{CaPa}$ - 6 de ellos operados con éxito después de fracaso de terapia de prueba, uno rechazó la cirugía. La única alternativa benigna no respondedora sería la pancreatitis del surco ${ }^{9}$, cuya terapia quirúrgica es una conducta frecuentemente necesaria. Efectivamente, en nuestros 3 casos con fracaso de tratamiento de prueba con prednisona, el diagnóstico definitivo fue $\mathrm{CaPa}$, con resección exitosa en 2 de ellos, siendo un caso inoperable por metástasis peritoneal no detectada con las imágenes. El temor frente al tratamiento de prueba es perder el tiempo, perder la ocasión de la resección curativa. Sin embargo, la experiencia publicada no avala progresión tan rápida de la enfermedad. Además, se trata de un cáncer de evolución ya larga en el momento del diagnóstico, es poco probable que dos semanas adicionales priven al paciente de la posibilidad de cirugía curativa. Indudablemente existen formas poco diferenciadas y muy agresivas, sin embargo, en estos casos el pronóstico es muy malo y tampoco parece significativa la pérdida de dos semanas.

La decisión quirúrgica debe ser tomada en un tiempo razonable. El diagnóstico morfológico e histológico sigue siendo el gold standard, en particular para descartar malignidad. Sin embargo, su rendimiento - como se detalló arriba - no es óptimo. Además, el mejor método, la EUS y biopsia no está considerada en el sistema chileno de seguros de salud, por consiguiente, su precio es alto y su accesibilidad está muy limitada. El tratamiento de prueba con control de imagen en 2 semanas es una herramienta útil en aclarar la naturaleza de la lesión: mejoría evidente comprueba PAI y evita la operación de una enfermedad tratable en forma conservador. Ausencia de respuesta obliga 
tomar una decisión quirúrgica, sin perjudicar al paciente, sin perder la posibilidad de resección de la lesión maligna.

En conclusión: El diagnóstico diferencial entre formas focales de PAI y CaPa generalmente es posible con sensibilidad y especificidad aceptable por los criterios clínicos, serológicos y -en primer lugar- por imágenes, hasta toma de biopsia. Sin embargo, en algunos casos el diagnóstico queda dudoso. En estos casos seleccionados el tratamiento de prueba con prednisona es muy útil y recomendable, siempre cuando la respuesta clínica y morfológica esté evaluada en 2 semanas: la mejoría es significativa en todos los casos de PAI, mientras ausencia de respuesta es prácticamente sinónimo de cáncer. La terapia de prueba es factible en cualquiera institución, no requiere tecnología adicional. La respuesta debe ser evaluada en el plazo corto establecido, sin perder más tiempo y por especialistas entrenados en diagnóstico y manejo de enfermedades pancreáticas.

Agradecimiento: A Juan Carlos Weitz, por sus comentarios y discusión del manuscrito.

\section{Referencias}

1. Sarles H, Sarles JC, Muratore R, Guien C. Chronic inflammatory sclerosis of the pancreas: an autonomous pancreatic disease? Am J Dig Dis 1961; 6: 688-98.

2. Yoshida K, Toki F, Takeuchi T, Watanabe S, Shiratori K, Hayashi N. Chronic pancreatitis caused by an autoimmune abnormality. Proposal of the concept of autoimmune pancreatitis. Dig Dis Sci 1995; 40: 1561-8.

3. Zhang L, Chari S, Smyrk TC, Deshpande V, Klöppel G, Kojima M, et al. Autoimmune Pancreatitis (AIP) Type 1 and Type 2. An International Consensus Study on Histopathologic Diagnostic Criteria. Pancreas 2011; 40: 1172-9.

4. Berger Z. Pancreatitis autoinmune: sospecha, diagnóstico diferencial y manejo. Gastroent Latinoam 2013 24/ supl. No1, 107-11.

5. Frulloni L, Scattolini C, Falconi M, Zamboni G, Capelli P, Manfredi R, et al. Autoimmune pancreatitis: differences between the focal and diffuse forms in 87 patients. Am J Gastroenterol 2009; 104: 2288-94.

6. Frulloni L, Amodio A, Katsotourchi AM, Vantini I. A practical approach to the diagnosis of autoinmune pancreatitis. World J Gastroenterol 2011; 17: 2076-9.

7. Hardacre JM, Iacobuzio-Donahue CA, Sohn TA, Abra- ham SC, Yeo CJ, Lillemoe KD, et al. Results of pancreaticoduodenectomy for lymphoplasmacytic sclerosing pancreatitis. Ann Surg 2003; 237: 853-8.

8. Konstantinidis IT, Warshaw AL, Allen JN, Blaszkowsky LS, Castillo CF, Deshpande V, et al. Pancreatic ductal adenocarcinoma: is there a survival difference for $\mathrm{R} 1$ resections versus locally advanced unresectable tumors? What is a "true" R0 resection? Ann Surg 2013; 257: 7316.

9. Becker V, Mischke U. Groove pancreatitis. Int J Pancreatol 1991; 10: 173-82.

10. Muñoz S, Mancilla C, Moyano L, Castillo C, Rossi R, Brahm J, et al. Pancreatitis autoinmune: Experiencia clínica y revisión de la literatura. Rev Med Chile 2010; 138: 295-302.

11. Ghazale A, Chari ST, Smyrk TC, Levy MJ, Topazian $\mathrm{MD}$, Takahashi N, et al. Value of serum IgG4 in the diagnosis of autoimmune pancreatitis and in distinguishing it from pancreatic cancer. Am J Gastroenterol 2007; 102: 1646-53.

12. Hamano H, Kawa S, Horiuchi A, Unno H, Furuya N, Akamatsu T, et al. High serum IgG4 concentrations in patients with sclerosing pancreatitis. N Engl J Med 2001; 344: 732-8.

13. Tabata T, Kamisawa T, Takuma K, Egawa N, Setoguchi K, Tsuruta K, et al. Serial Changes of Elevated Serum IgG4 Levels in IgG4-related Systemic Disease. Intern Med 2011; 50: 69-75.

14. Lévy P, Hammel P, Ruszniewski P. Diagnostic challenge in autoimmune pancreatitis: beware of shipwreck! Gut 2008; 57: 1646-7.

15. Levy MJ. Endoscopic ultrasound-guided trucut biopsy of the pancreas: prospects and problems. Pancreatology 2007; 7: 163-6.

16. Berger Z, Bufadel ME, Navarro A. Endoscopic methods in the diagnosis and treatment of pancreatic cancer. J Cancer Therapy 2013; 4: 1-6.

17. Chari ST, Smyrk TC, Levy MJ, Topazian MD, Takahashi $\mathrm{N}$, Zhang L, et al. Diagnosis of autoimmune pancreatitis: the Mayo Clinic experience. Clin Gastroenterol Hepatol 2006; 4: 1010-6.

18. Matsubayashi $\mathrm{H}$, Tomita $\mathrm{H}$, Sugiura $\mathrm{T}$, Sasaki K, Inui $\mathrm{T}$, Takizawa K, et al. Autoimmune Pancreatitis without a Response to Steroid Therapy: A Case which Met Criteria after Withdrawal of Steroid. Inter Med 2009; 48: 208791.

19. Moon SH, Kim MH, Park DH, Hwang CY, Park SJ, Lee SS, et al. Is a 2-week steroid trial after initial negative investigation for malignancy useful in differentiating autoimmune pancreatitis from pancreatic cancer? A prospective outcome study. Gut 2008; 57: 1704-12. 